\title{
Efetividade de exercícios físicos domiciliares: uma intervenção sobre o desempenho físico em mulheres idosas
}

Effectiveness of home physical exercise: an intervention on physical exercise in elderly women

\author{
Maria da Conceição Lopes Ribeiro ${ }^{1}$ \\ Letícia Peres Luiz ${ }^{2}$ \\ Joilson Meneguci ${ }^{2}$ \\ Jair Sindra Virtuoso Junior ${ }^{2}$ \\ Sheilla Tribess ${ }^{2, *}$
}

\section{Resumo}

Objetivo: analisar a efetividade da intervenção comunitária com a utilização do guia domiciliar de exercícios físicos nas variáveis da aptidão funcional. Métodos: participaram do estudo 91 mulheres com idade $\geq 61$ anos, divididas em grupo intervenção $(n=49)$ e controle $(n=42)$. As idosas realizaram testes de desempenho físico antes e após 12 semanas de intervenção. A intervenção foi realizada com o uso de um guia domiciliar de exercícios físicos associados a estratégias motivacionais (ligações telefônicas e visitas domiciliares). Utilizaram-se procedimentos da estatística descritiva e inferencial (teste de quiquadrado, teste $t$ de student para amostras independentes e ANOVA de medidas repetidas, $p<0,05$ ). Resultados: ao final da intervenção as idosas do Gl apresentaram melhorias para as variáveis de resistência de membros superiores e inferiores e resistência aeróbia. Conclusão: a intervenção domiciliar de baixa interação com o uso de guia de exercícios físicos teve impacto positivo na saúde das idosas.
\end{abstract}

Palavras-chave:exercício físico, idoso, estudos de intervenção.

\section{Abstract}

Objective: this study aims to analyze the effectiveness of home-based exercise guide in physical performance variables in older women. Methods: participated in the study 91 women aged $\geq 61$ years, divided in intervention group $(n=49)$ and control group $(n=42)$. The older women were underwent physical performance tests before and after 12 weeks of intervention. The intervention was realized using the home based guide of physical exercises associated with motivational strategies (phone calls and home visits). For data analysis, were used procedures of descriptive statistics and inferential (chi-square test, test $t$ of student to independent samples and ANOVA of repeated measures, $\mathrm{p}<0.05$ ). Results: at the end of 12 weeks of intervention, it was verified that the intervention group showed improvements of resistance variables of upper and lower limbs and aerobic endurance. Conclusion: the home based intervention using the physical exercises guide had positive impact on the health of the elderly.
\end{abstract}

Keywords: exercise, elderly, intervention studies.
Afiliação dos autores

${ }^{1}$ Universidade Federal do Triângulo Mineiro, Uberaba, Minas Gerais,

Brasil.

'Universidade Federal do Piauí,

Teresina, Piauí, Brasil

*Autor correspondente

Departamento de Ciências do Esporte, Av. Getúlio Guaritá, 159 Centro Educacional, Sala 333-E, Abadia, CEP 38025-440, Uberaba, MG

e-mail: sheilla.tribess@uftm.edu.br

Conflito de interesses

Os autores declararam não haver conflito de interesses.

Processo de arbitragem

Recebido: 10/02/2018 Aprovado: 15/03/2018 


\section{Introdução}

Nas últimas décadas têm sido observado contínuo aumento da população idosa, fato este que paralelamente tem contribuído para o aumento da prevalência de doenças crônicas não transmissíveis, a exemplo do diabetes, dislipidemias e da hipertensão arterial sistêmica, além do declinio da força e massa muscular ${ }^{1}$. Essas doenças alteram os níveis sanguíneos séricos de glicose e perfil lipídico ${ }^{2,3}$ e podem conduzir o organismo a um estado pró-inflamatório.

É bem afirmado na literatura os benefícios do exercício para idosos ${ }^{4,5,6}$ e a relação entre comprometimento, limitação funcional e incapacidade ${ }^{7}$, o exercício tem o potencial de retardar ou moderar a incapacidade. De fato, há achados na literatura para isso, pelo menos entre aqueles que já estão fisicamente frágeis ${ }^{8,9,10}$. No entanto ainda não está claro se o exercício pode atrasar o início da incapacidade em pessoas idosas ainda ativas ${ }^{11,12}$

Nesse contexto e frente às novas estatégias de intervenções ${ }^{5,6,13,14,15}$, uma maneira nova e economicamente viável para promover a atividade física em idosos é através das chamadas intervenções não face-a-face, que são caracterizadas como intervenções de interação reduzida do participante com o prestador da intervenção ${ }^{16}$.

A utilização do guia de exercícios domiciliares é uma proposta de intervenção possui benefícios administrativos, logísticos devido à facilidade de divulgação e sensibilização dos usuários. Os exercícios físicos contidos no guia são comuns as atividades do cotidiano do idoso e visam proporcionar melhorias os componentes da aptidão física e consequentemente a saúde do idoso ${ }^{17,18}$.

Assim, o estudo teve como objetivo analisar a efetividade do uso do guia domiciliar de exercícios físicos nas variáveis de desempenho físico em mulheres idosas.

\section{Métodos}

Trata-se de estudo quase-experimental desenvolvido com idosas residentes na cidade de Uberaba, MG. Para o estudo foram recrutadas 104 idosas com idade entre 60 e 80 anos cadastradas em cinco Unidades Básicas de Saúde circunvizinhas a sede do Programa de Pós-Graduação em Educação Física da Universidade Federal do Triângulo Mineiro (PPGEF-UFTM)

Os critérios de inclusão para participar da pesquisa foram: não realizar nenhuma atividade física ou exercício físico de lazer; possuir telefone fixo ou celular; não apresentar dificuldades na acuidade visual e auditiva em grau severo; não fazer uso de cadeiras de rodas; não estar acamado provisório ou definitivo; não possuir sequelas graves de acidente vascular encefálico (AVE) com perda localizada de força; e não possuir doença em estágio terminal.

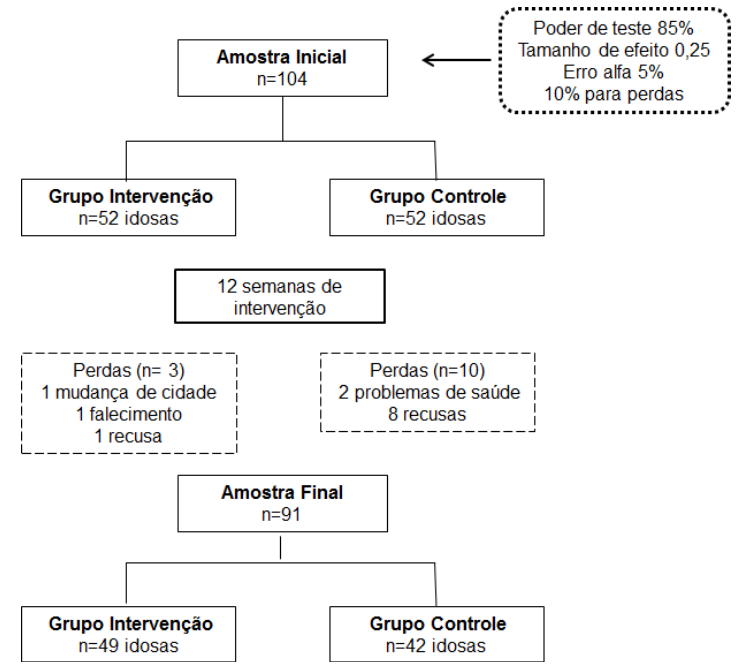

Figura 1. Organograma da seleção das idosas participantes do estudo de intervenção com uso do Guia Domiciliar de Exercícios Físicos. UberabaMG.
Instrumento para Coleta de Dados

As idosas participantes do estudo responderam um questionário, aplicado em forma de entrevista individual e realizaram os testes de desempenho físico antes e a após 12 semanas de intervenção. As informações coletas foram:

Informações Sociodemográficas: idade, estado civil, escolaridade, situação ocupacional e arranjo familiar.

Indicadores de Saúde: presença de doenças autorreferidas (presença ou ausência de doenças); uso de medicamentos (quantidade de medicamentos consumidos diariamente); ocorrência de quedas no último ano; histórico de hospitalização nos últimos seis meses; consumo de tabaco e de bebidas alcoólicas.

Sintomatologia Depressiva: avaliada pela versão reduzida da Escala de Depressão Geriátrica (GDS-15) validada para população brasileira por Almeida e Almeida19, sendo considerado para 0 presente estudo a presença de sintomatologia depressiva indivíduos com pontuação igual ou superior a 5 pontos.

Função Cognitiva: avaliada pelo Mini Exame do Estado Mental (MEEM), desenvolvido por Folstein, Folstein e Mchugh ${ }^{20}$. Devido a influência do nível de escolaridade no MEEM, utilizouse a versão brasileira de Almeida21, cujos pontos de corte para a presença do déficit cognitivo levam em consideração os anos de estudo, sendo para os indivíduos sem escolaridade $\leq 19$ pontos; indivíduos com escolaridade $\leq 23$ pontos.

Atividade Física Habitual: avaliada pelo Questionário Internacional de Atividades Físicas adaptado para idosas ${ }^{22}$. Para o presente estudo utilizou-se somente o domínio de atividade física de lazer com critério de inclusão para participação na intervenção.

Aptidão Funcional: analisada pelos testes de desempenho físico da bateria de testes Fullerton proposto por Rikli e Jones ${ }^{22}$ A bateria é composta pelos testes de flexibilidade de membros inferiores (teste sentar e alcançar) e superiores (teste de mãos nas costas); força e resistência muscular localizada para membros inferiores (teste de sentar e levantar da cadeira no qual se pontua o número de repetições completas durante 30 segundos) e para membros superiores (teste de flexão de cotovelo com número de repetições completas durante 30 segundos), resistência aeróbica (teste de marcha estacionária de 2 minutos, computa-se o número de passadas completas) e agilidade e equilíbrio dinâmico (teste de ir e vir de 2,44m, tempo em segundos para completar o percurso)

Por questões de segurança na realização dos testes de desempenho físico a pressão arterial da idosa foi aferida após cinco minutos de repouso e antes da realização dos testes por meio de um esfigmomanômetro digital - Omron HEM 710INT. Caso a pressão arterial sistólica apresentasse valores $\geq 140$ $\mathrm{mm} / \mathrm{Hg}$, e/ou os valores da pressão diastólica $\geq 90 \mathrm{~mm} / \mathrm{Hg}$ os testes de desempenho físico não foram realizados.

\section{Protocolo de Intervenção}

As idosas do grupo intervenção receberam o kit de exercícios físicos para ser utilizado durante as 12 semanas da intervenção, enquanto que as idosas do grupo controle foram orientadas a manter a sua rotina habitual e foram acompanhadas por ligações telefônicas mensais.

O kit de exercícios físicos foi composto pelo guia domiciliar de exercícios físicos (um folheto com imagens e descrição dos exercícios físicos realizados pelas idosas), um diário mensal para anotar os dias e a satisfação com a prática dos exercícios físicos, duas bolas de borracha pequenas de $5 \mathrm{~cm}$ para trabalhar a força e resistência dos membros superiores, uma corda de um metro para auxiliar nos exercícios de alongamento e um jogo de memória de figuras com imagens de animais, personalidade da televisão, cores, frutas, meios de transporte ou verduras.

Os exercícios físicos descritos no guia domiciliar referem-se a exercícios para incremento de força e equilíbrio (realizado de 2 a 4 vezes por semana), resistência aeróbia ( 4 a 7 vezes na semana), flexibilidade para membros superiores e inferiores (diariamente) e jogos para a memória (diariamente).

Durante as 12 semanas da intervenção, o grupo intervenção recebeu 11 visitas domiciliares e cinco ligações telefônicas para reforçar a importância da utilização do guia domiciliar de exercícios físicos e estimular a aderência ao programa de intervenção (Figura 2) 


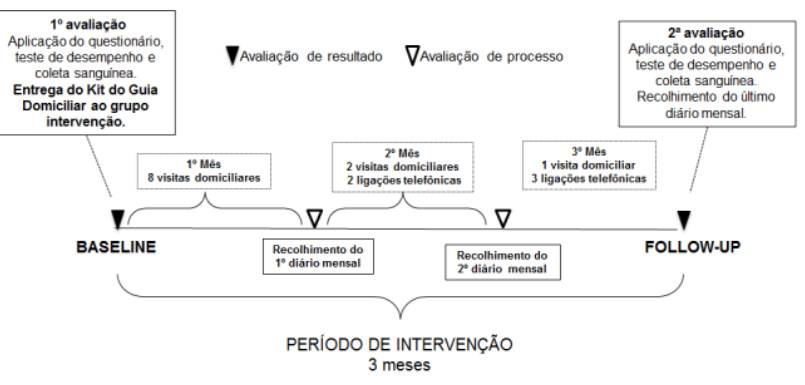

Figura 2. Fluxograma da intervenção e das avaliações de resultado e de processo da intervenção com mulheres idosas.

\section{Cuidados Éticos}

O presente projeto foi submetido à apreciação do Comitê de Ética e Pesquisa da Universidade Federal do Triângulo Mineiro e aprovado pelo parecer ํㅜ 712467/2014. As idosas que aceitaram participar do estudo assinaram o Termo de Consentimento Livre Esclarecido.

\section{Análise dos Dados}

Para tabulação dos dados foi utilizado o software Epidata 3.1 e a análise por meio do software StatisticalPackage for the Social Sciences(SPSS), versão 21.0. Para analisar a efetividade da intervenção nas variáveis de desempenho físico e bioquímicas inicialmente realizou-se o teste de Shapiro Wilk para verificar a normalidade dos dados. Após a análise de normalidade utilizou-se 0 escore $Z$ para as variáveis que rejeitaram a hipótese de distribuição normal. Com a padronização dos dados, utilizou-se $o$ teste $t$ de student para amostras independentes no momento baseline para comparação do grupo controle e grupo intervenção. Para análise de interação dos grupos e momentos foi realizado o teste de ANOVA de medidas repetidas para as variáveis que não apresentaram diferenças no momento baseline entre os grupos. Para as variáveis que apresentaram diferenças, foram calculados os valores de delta $(\Delta)$ e posteriormente realizado o teste t student para amostras independentes.

Para todas as análises realizadas adotou-se o nível de significância de $5 \%$.

\section{Resultados}

As 91 idosas que finalizaram a intervenção tinham entre 61 e 80 anos de idade, com média de idade de 70,11 anos $(\mathrm{DP}=4,70)$ e não apresentavam diferenças significativas entre os grupos para as variáveis sociodemográficas e de saúde.

De forma geral, as idosas estavam concentradas na faixa etária de 70 a 80 anos (56\%), eram viúvas $(42,9 \%)$ ou casadas/vivendo com parceiros $(36,2 \%)$, residentes em domicílios multigeracionais $(46,2 \%)$, especificamente bigeracionais (24,2\%, avós e filhos), apresentavam baixo nível educacional, entre um e quatro anos de estudos $(76,9 \%), 61,6 \%$ eram aposentadas ou pensionistas.

As idosas, em sua maioria, relataram a presença de doenças $(97,8 \%)$ e o uso de medicamentos de forma continua $(90,1 \%)$. Nos últimos seis meses, $14,3 \%$ das idosas foram hospitalizadas e $29,7 \%$ relataram a ocorrência de quedas no último ano. A presença de sintomas depressivos foi percebida em $38,5 \%$ das idosas e a presença de declínio cognitivo em $65,9 \%$. Em relação às variáveis comportamentais tabagismo e consumo de álcool foram relatado respectivamente por $23,1 \% \mathrm{e}$ $18,7 \%$ das idosas.

Para análise dos grupos (intervenção e controle) no baseline, 0 teste $t$ de student para amostra independente evidenciou que os dois grupos foram similares em todas as variáveis analisadas, exceto para as variáveis flexibilidade de membros superiores e colesterol total (Tabela 1).
Distribuição das variáveis de desempenho físico no momento pré intervenção de acordo com os grupos intervenção e controle.

\begin{tabular}{|c|c|c|c|c|c|c|}
\hline \multirow{3}{*}{ Variáveis Analisadas } & \multicolumn{4}{|c|}{ Grupos (n=91) } & \multirow[b]{3}{*}{ T } & \multirow[b]{3}{*}{$p^{*}$} \\
\hline & \multicolumn{2}{|c|}{ Intervenção } & \multicolumn{2}{|c|}{ Controle } & & \\
\hline & Média & $\overline{D P}$ & Média & $\overline{D P}$ & & \\
\hline \multicolumn{7}{|l|}{ Variáveis de Desempenho } \\
\hline Flexibilidade MS $(\mathrm{cm})$ & $-8,5$ & 9,1 & $-12,6$ & 8,6 & 2,191 & 0,031 \\
\hline Flexibilidade MI (cm) & 0,1 & 7,4 & $-2,1$ & 5,9 & 1,508 & 0,135 \\
\hline US (repetições) & 11,6 & 2,9 & 11,6 & 3,6 & 0,0 & 0,996 \\
\hline Resistência MI (repetições) & 9,6 & 2,8 & 8,9 & 3,3 & 1,258 & 0,212 \\
\hline Agilidade (segundos) & 9,7 & 2,8 & 10,0 & 2,8 & 0,480 & 0,632 \\
\hline Resistência Aeróbia & 79,0 & 18,7 & 76,9 & 20,0 & 0,510 & 0,611 \\
\hline
\end{tabular}

*Teste $\mathrm{t}$ de Student para amostras independentes; MS=membros superiores; $\mathrm{Ml}=$ membros inferiores.

A ANOVA de medidas repetidas apontou interação entre grupos e momentos, para as variáveis: resistência de membros superiores e inferiores, resistência aeróbia e glicemia, ou seja, os grupos se comportaram de maneiras diferentes entre os momentos pré e pós intervenção (Tabela 2).

\section{Tabela 2}

Distribuição das variáveis de desempenho nos momentos pré e pós intervenção de acordo com os grupos intervenção e controle.

\begin{tabular}{|c|c|c|c|c|c|c|}
\hline & \multicolumn{6}{|c|}{ Grupo Intervenção } \\
\hline Variáveis & \multicolumn{2}{|c|}{ Pré } & \multicolumn{2}{|c|}{ Pós } & \multirow[b]{2}{*}{$\mathrm{F}$} & \multirow[b]{2}{*}{$p^{*}$} \\
\hline Desempenho & Média & DP & Média & DP & & \\
\hline Flexibilidade MI & 0,1 & 7,4 & 1,3 & 6,3 & 0,989 & 0,323 \\
\hline Resistência MS & 11,6 & 2,9 & 14,0 & 3,4 & 7,929 & 0,006 \\
\hline Resistência MI & 9,6 & 2,8 & 11,8 & 3,6 & 5,401 & 0,022 \\
\hline Agilidade & 9,7 & 2,8 & 9,1 & 3,0 & 0,027 & 0,869 \\
\hline $\begin{array}{l}\text { Resistência } \\
\text { Aeróbia }\end{array}$ & 79,0 & 18,7 & 92,3 & 25,3 & 10,975 & 0,001 \\
\hline \multicolumn{7}{|c|}{ Grupo Controle } \\
\hline Variáveis & \multicolumn{2}{|c|}{ Pré } & \multicolumn{2}{|c|}{ Pós } & & \\
\hline Desempenho & Média & DP & Média & $\mathrm{DP}$ & $\mathrm{F}$ & $p^{*}$ \\
\hline Flexibilidade MI & 5,9 & $-2,6$ & 7,9 & 5,9 & 0,989 & 0,323 \\
\hline Resistência MS & 3,6 & 11,9 & 3,3 & 3,6 & 7,929 & 0,006 \\
\hline Resistência MI & 3,3 & 9,3 & 3,2 & 3,3 & 5,401 & 0,022 \\
\hline Agilidade & 2,8 & 9,5 & 3,1 & 2,8 & 0,027 & 0,869 \\
\hline Resistência & 20,0 & 72,9 & 20,0 & 20,0 & 10,975 & 0,001 \\
\hline
\end{tabular}

Aeróbia

${ }^{*}$ ANOVA para medidas repetidas; $\mathrm{MS}=$ membros superiores; $\mathrm{Ml}=$ membros inferiores.

O grupo intervenção apresentou melhoras no desempenho da resistência de membro superior, resistência de membro inferior e resistência aeróbia, enquanto que, no grupo controle ocorreu uma diminuição da resistência aeróbia e uma manutenção no desempenho da resistência de membros superiores e inferiores (Figura 3).

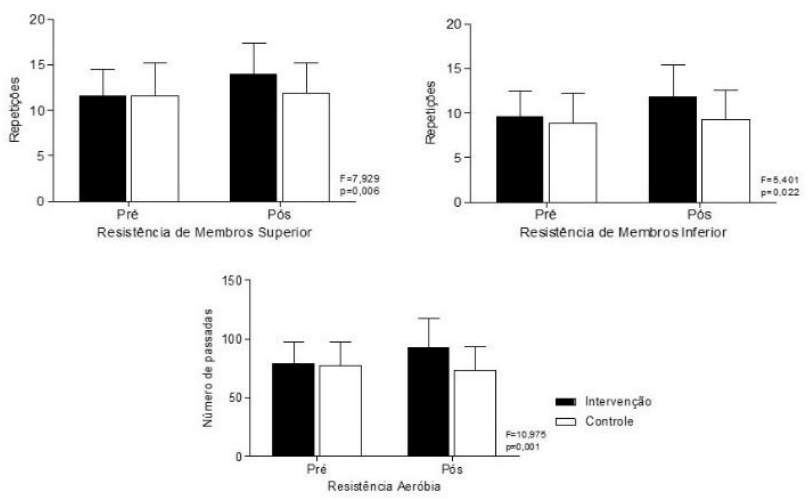

Figura 3.Resistência de membros superiores, inferiores e aeróbia nos momentos pré e pós intervenção para os grupos intervenção e controle.

Para a variável flexibilidade de membros superiores foi calculado o valor de delta $(\Delta)$ entre os momentos pré e pós intervenção pelo fato dos grupos intervenção e controle terem se comportado de maneira diferente no baseline.

$O$ teste $t$ de student para amostras independentes indicou diferenças significativas ( $t=-4,877$ e $p=0,000$; ) entre os grupos para $\Delta$ colesterol total, sendo que o grupo intervenção apresentou uma redução dos valores de colesterol total (média $=-21,7$ e $\mathrm{DP}=49,6$ ), enquanto que no grupo controle esses valores elevaram-se (média=23,6 e DP=36,7). 
$\mathrm{O}$ teste $\mathrm{t}$ de student para amostras independentes não indicou diferenças significativas para $\Delta$ flexibilidade de membros superiores $(t=1,579$ e $p=0,118)$ entre os grupos, ou seja, os grupos mantiveram os valores da flexibilidade de membros superiores após 12 semanas de intervenção.

\section{Discussão}

Após 12 semanas de intervenção, observou-se no presente estudo a efetividade de um guia domiciliar de exercícios físicos no desempenho físico da resistência de membros superiores, inferiores e aeróbia. Esses achados justificam e confirmam a importância de intervenções com interacõos reduzidas e realizadas a partir de exercício físico de baixa intensidade e de fácil aplicação para idosos

A possibilidade de se obter resultados positivos com exercícios realizados no domicilio é também foi verificada em uma intervenção, após 12 semanas possível atenuar a fragilidade na mobilidade de idosos participantes do programa ${ }^{10}$.

A efetividade de intervenções a partir da entrega de kits que encorajam a prática de exercícios físicos, e proporciona benefícios à saúde tem sido visto em estudos prévios realizados em países desenvolvidos, entretanto estes estudos não avaliaram os resultados de desempenho físico dos participantes ${ }^{14,23,24}$.

A melhora no desempenho físico é de fundamental importância para idosos, uma vez que o declínio dos componentes da aptidão física associa-se negativamente à saúde, levando a incapacidade funcional, morbidades e mortalidade $^{7,25}$.

Uma das consequências do envelhecimento é diminuição da massa magra, essa diminuição da resistência muscular influencia a qualidade muscular, associando-se ao desenvolvimento de processos incapacitantes, podendo levar o indivíduo a condição de dependência, fragilidade e mortalidade ${ }^{26,27}$. O comportamento sedentário e a inatividade física estão entre os fatores que contribuem para a perda da resistência muscular no idoso ${ }^{27,28,28}$.

No presente estudo, foi identificado que o grupo participante da intervenção apresentou melhor desempenho na resistência de membros superiores entre os momentos, o que pode proporcionar a capacidade de manter esforços repetitivos por um maior período de tempo, contribuindo para execução de maneira mais efetiva das atividades do cotidiano e para a mobilidade do idoso ${ }^{30,31}$.

A qualidade de execução de atividades do cotidiano, como carregar pesos, subir escadas e levantar-se proporciona autonomia funcional ao idoso, além de atuar na prevenção de quedas, fraturas e hospitalizações ${ }^{5,32}$

Os exercícios para membros inferiores propostos na presente intervenção, mesmo não utilizando cargas externas foram efetivos no incremento de resistência para membros inferiores. A diminuição do desempenho motor durante o envelhecimento é específico à tarefa, determinado de maneira seletiva pelo desuso de determinadas funções ${ }^{33}$. Os exercícios similares às funções do cotidiano podem ser utilizados como estratégia de fácil aderência e de amplo alcance populacional.

A melhora da resistência nos membros inferiores do grupo intervenção pode justificar a alteração positiva que ocorreu na resistência aeróbia. A diminuição da força de membros inferiores se relacionada a prejuízos no padrão da marcha e de atividades funcionais ${ }^{31,34}$

O componente de aptidão cardiorrespiratório começa a ter uma redução ao avançar da idade, pois em seu mecanismo ocorre diminuição nos valores do débito cardíaco, contratibilidade do miocárdio, limitação da função pulmonar pela diminuição alveolar, aumento da resistência vascular periférica e, consequentemente, a elevação da pressão arterial $\left.\right|^{35,36}$

Em indivíduos idosos, a capacidade cardiorrespiratória quando treinada foi eficaz na redução dos fatores de risco cardiovascular dos idosos sedentários e hipertensos estudado $^{36}$. Ainda, a participação em programas de exercícios associa-se a uma alteração na reserva cardiovascular e adaptações musculoesqueléticas que capacitam os idosos treinados a sustentarem uma carga de exercício com menor estresse cardiovascular, menor fadiga muscular e garantir melhores níveis dos componentes da aptidão física ${ }^{37}$.

O engajamento na intervenção domiciliar do presente estudo proporcionou melhora na resistência aeróbia quando comparado com o grupo não participante, o que pode contribuir para mobilidade funcional ${ }^{38}$.

A partir das evidências apontadas, a intervenção desenvolvida no presente estudo pode ser considerada uma estratégia para atenuação do processo de dependência na realização de atividades da vida diária dos idosos, devido ao aparecimento das dificuldades no desempenho físico que parecem anteceder aos processos incapacitantes ${ }^{39}$.

Os pontos fortes desde estudo foram: a intervenção ter sido realizada no ambiente domiciliar, o que pode encorajar e facilitar o engajamento dos idosos a prática do exercício físico; a elaboração do guia e seus materiais correspondentes serem de baixo custo tornando a intervenção viável de ser generalizada; $e$ por fim, o fato de um programa de 12 semanas, tendo como estratégia a recomendação de exercícios simples de baixa intensidade e de baixa interação ter sido capaz de promover modificações em alguns componentes da aptidão física.

As limitações do estudo referem-se a perda de alguns participantes do estudo durante a intervenção, o não controle da dieta e medicamentos das participantes; o período de 12 semanas, que pode não ter sido suficiente para proporcionar alterações significativas em algumas variáveis do desempenho físico e a amostra ter sido composta exclusivamente por mulheres.

\section{Conclusão}

Pode-se concluir que a intervenção domiciliar realizada por 12 semanas com baixa interatividade mostrou-se efetiva para atenuar as limitações funcionais ao apresentar resultados positivos em alguns componentes do desempenho físico como na resistência muscular de membros superiores e inferiores e na resistência aeróbia. As informações do presente estudo fornecem subsídios para o desenvolvimento de estratégias de intervenções simples e acessíveis, pois quando estas são direcionadas da maneira correta, planejada e executada, resultam em melhoras efetivas. Espera-se que este estudo possa fornecer informações para os órgãos públicos de saúde, podendo ser considerado um modelo a ser seguido nas unidades básicas de saúde.

\section{Agradecimentos}

Os autores agradecem aos integrantes do Núcleo de Pesquisa em Atividade Física e Saúde (NEAFISA/UFTM) pela colaboração na coleta de dados.

\section{Financiamento}

Fundação de Amparo à Pesquisa do Estado de Minas Gerais - FAPEMIG (APQ-01023-14).

Coordenação de Aperfeiçoamento de Pessoal de Ensino Superior - CAPES (bolsa DS/CAPES - Mestrado).

\section{Referências}

1. Bherer L, Erickson KI, Liu-Ambrose T. A Review of the effects of physical activity and exercise on cognitive and brain functions in older adults. $J$ Aging Res 2013; 1(1):1-8.

2. Tan YY, Gast GC, Van der Schouw YT. Gender differences in risk factors for coronary heart disease. Maturitas 2010; 1(65):149-160.

3. Ribeiro AS, Tomeleri CM, Souza MF, Pina FLC, Schoenfeld BJ, Nascimento MA, Venturini D, Barbosa D, Cyrino ES. Effect of resistance training on C-reactive protein, blood glucose and lipid profile in older women with differing levels of RT experience. Age (Dordr) 2015; 37(6):109.

4. Hill KD, Hunter SW, Batchelor FA, Cavalheri V, Burton E. Individualized home-based exercise programs for older people to reduce falls and improve physical performance: A systematic review and meta-analysis. Maturitas 2015; 82(1):72-84

5. Duckham RL, Masud T, Taylor R, Kendrick D, Carpenter H, lliffe S, Morris $\mathrm{R}$, Gage H, Skelton DA, Dinan-Young S, Brooke-Wavell K. Randomised controlled trial of the effectiveness of community group and home-based falls prevention exercise programmes on bone health in older people: the ProAct65+ bone study. Age Ageing 2015; 44(4):573-9.

6. Sosnoff JJ, Finlayson M, McAuley E, Morrison S, Motl RW. Home-based exercise program and fall-risk reduction in older adults with multiple sclerosis: phase 1 randomized controlled trial. Clin Rehabil 2014; sclerosis: phas

7. Verbrugge L, Jette A. The disablement process. Soc Sci Med 1994 1(38):1-14.

8. Giné-Garriga $M$, Roqué-Fíguls $M$, Coll-Planas L, Sitjà-Rabert $M$, Salvà $A$ Physical exercise interventions for improving performance-based measures 
of physical function in community-dwelling, frail older adults: a systematic review and meta-analysis. Arch Phys Med Rehabil 2014 95(4):753-769.

9. Fairhall N, Sherrington C, Lord SR, Kurrle SE, Langron C, Lockwood K Monaghan N, Aggar C, Cameron ID. Effect of a multifactorial, interdisciplinary intervention on risk factors for falls and fall rate in frail older people: a randomised controlled trial. Age Ageing 2014 43(5):616-22.

10. Clegg A, Barber S, Young J, lliffe S, Forster A. The Home-based Older People's Exercise (HOPE) trial: a pilot randomised controlled trial of a home-based exercise intervention for older people with frailty. Age Ageing 2014; 43(5): 687-95.

11. Motl RW, McAuley E. Physical activity, disability and quality of life in older adults. Phys Med Rehabil Clin N Am. 2010; 1(21): 299-308.

12. Day L, Hill KD, Jolley D, Cicuttini F, Flicker L, Segal L. Impact of Tai Chi on Impairment, Functional Limitation, and Disability Among Preclinically Disabled Older People: A Randomized Controlled Trial . Arch Phys Med Rehabil 2012; 93(8): 1400-1407.

13. Pfaff JJ, Alfonso $\mathrm{H}$, Newton RU, Sim M, Flicker L, Almeida OP. ACTIVEDEP: a randomised, controlled trial of a home-based exercise intervention to alleviate depression in middle-aged and older adults. $\mathrm{Br} J$ Sports Med. 2014;48(3):226-32.

14. Asmidawati A, Hamid TA, Hussain RM, Hill KD. Home based exercise to improve turning and mobility performance among community dwelling older adults: protocol for a randomized controlled trial. BMC Geriatr 2014; $8(14): 100$.

15. Müller AM, Khoo S, Morris T. Text Messaging for Exercise Promotion in Older Adults From an Upper-Middle-Income Country: Randomized Controlled Trial. J Med Internet Res 2016; 18(1):e5.

16. Müller AM, Khoo S. Non-face-to-face physical activity interventions in older adults: a systematic review. Int J BehavNutr Phys Act 2014; 11(1):35

17. Vagetti GC, Barbosa Filho VC, Moreira NB, de Oliveira V, Mazzardo O, de Campos W. The prevalence and correlates of meeting the current physical activity for health guidelines in older people: a cross-sectional study in Brazilian women. ArchGerontolGeriatr 2013;56(3):492-500.

18. Honório G, Martins $\mathrm{H}$, Basso J, Alvarez Â, Meirelles B, Santos S. Estratégias de promoção da saúde dos idosos no Brasil: um estudobibliométrico. RevistaEnfermagem da UERJ 2013; 21(1):121-126.

19. Almeida OP, Almeida SA. Confiabilidade da versãobrasileira da escala de depressãoemgeriatria (GDS) versãoreduzida. Arquivos de Neuropsiquiatria 1999; 57(3): 421-426.

20. Folstein MF, Folstein SE, Mchugh PR "Mini-mental state". A practical method for grading the cognitive state of patients for the clinician. Journal of Psychiatric Research 1975; 12(3): 189-198.

21. Almeida OP. Mini exame do estado mental e o diagnóstico de demência no Brasil. Arquivos de Neuropsiquiatria1998; 56(3): 605-612.

22. Benedetti, T. B., Mazo, G. Z. \& Barros, M. V. G. (2004). Aplicação do questionáriointernacional de atividadesfísicas para avaliação do nível de atividadesfísicas de mulheresidosas: validadeconcorrente reprodutibilidade teste-reteste. RevistaBrasileira de Ciência e Movimento, reprodutibilidade teste-reteste. RevistaBrasileira de Ciência e Movimento,
$12(1), 25-34$.

22. Rikli RE, Jones CJ. Development and validation of a functional fitness test for community-residing older adults. J Aging Phys Act 1999; 7(2):129-61.

23. Geraedts HA, Zijlstra W, Zhang W, Bulstra S, Stevens M. Adherence to and effectiveness of an individually tailored home-based exercise program for frail older adults, driven by mobility monitoring: design of a prospective cohort study. BMC Public Health 2014; 7(14):570.
24. Burke L, Lee AH, Jancey J, Xiang L, Kerr DA, Howat PA, Hills AP, Anderson AS. Physical activity and nutrition behavioural outcomes of a home-based intervention program for seniors: a randomized controlled trial. Int J BehavNutr Phys Act 2013;10(14):1-8.

25. Den Ouden ME, Schuurmans MJ, Arts IE, van der Schouw YT. Physical performance characteristics related to disability in older persons: A systematic review. Maturitas 2011; 69(3):208-19.

26. Brito CJ, Volp ACP, Nóbrega OT, Silva Júnior FL, Mendes, EL, Roas AFCM, Barros JF, Córdova C. Exercício físico como fator de prevenção aos processos inflamatórios decorrentes do envelhecimento. Motriz 2011; 17(3): 544-555.

27. da Silva VD, Tribess S, Meneguci J, Sasaki JE, Santos DAT, Carneiro JAO, Virtuoso Júnior JS. Time Spent in Sedentary Behaviour as Discriminant Criterion for Frailty in Older Adults. Int $\mathrm{J}$ Environ Res Public Health 2018; 15(7): 1336 .

28. Hamer M, Stamatakis E. Screen-based sedentary behavior, physical activity, and muscle strength in the English longitudinal study of ageing. PLoS One 2013; Jun 3;8(6):e66222.

29. Roma MFB, Busse AL, Betoni RA, Melo AC, Kong J, Santarem JM, Jacob $W$. Efeitos das atividades físicas resistidas e aeróbia em idosos em relação à aptidão física e à funcionalidade: ensaio clínico prospectivo. Einstein 2013; 11(2):153-157.

30. Barbosa A R, Souza JMP, Lebrão ML, Marucci MFN. Relação entre estadonutricional e força de preensão manual emidosos do município de São Paulo, Brasil. Rev Bras CineantropomDesempenho Hum 2006; 8(1):37-44.

31. Bez JPO, Neri AL. Velocidade da marcha, força de preensão e saúdepercebidaemidosos: dados da rede FIBRA. Ciência\&SaúdeColetiva 2014; 19(8):3343-3353

32. Lamboglia CG, Costa, RG, Franchi, KMB, Pequeno LL, Pinheiro MHNP. Efeito do tempo de prática de exercíciofísiconaaptidãofísicarelacionada à saúdeemmulheresidosas. RevistaBrasileiraemPromoção da Saúde 2014; 27(1): 29 .

33. Teixeira LA. Declínio de desempenho motor no envelhecimento é específico à tarefa. RevistaBrasileira de Medicina do Esporte 2006; 12(6) 351-355.

34. Pícoli T da S, Figueiredo LL de, Patrizzi LJ. Sarcopenia e envelhecimento. FisioterapiaemMovimento 2011; 24(3): 455-462

35. Fechine BRA, Trompieri N. O processo de envelhecimento: as principais alterações que acontecem com o idoso com o passar dos anos. InterscinecePlace 2012;1(20):106-132.

36. Hortencio MN da S, Silva JKS da, Zonta MA, Melo CPA de, França CN. Efeitos de exercíciosfísicossobrefatores de risco cardiovascular emidososhipertensos. RevistaBrasileira de Promoção da Saúde 2018; 31(2):1-9.

37. American College of Sports Medicine, Chodzko-Zajko WJ, Proctor DN, Fiatarone Singh MA, Minson CT, Nigg CR, Salem GJ, Skinner JS, American College of Sports Medicine position stand. Exercise and physical American College of Sports Medicine position stand. Exercise and physi

38. Santos AS, Tribess S, Pinto LLT, Ribeiro MCL, Rocha SV, Virtuoso Júnior JS. Velocidade de caminhadacomoindicador para aincapacidadefuncionalemidosos. Motricidade 2014:10(3):50-60.

39. Morey MC, Pieper CF, Cornoni-Huntley J.Physical fitness and functiona limitations in community-dwelling older adults. Med Sci Sports Exerc 1998; 30(5): $715-23$. 